\title{
Physical therapy rehabilitation following total shoulder replacement using total motion release techniques in combination with traditional intervention
}

\begin{abstract}
Aim: The incidence of shoulder replacement surgery has increased significantly in the past 10 years, becoming the 3rd most performed total joint arthroplasty after hip and knee and is expected to continue to rise with the increase in the active senior population of the United States. This case study describes rehabilitation following total shoulder arthroplasty (TSA) using a combination of traditional intervention and Total Motion Release (TMR) techniques. TMR is an intervention technique based on the concept of contralateral movement to affect fascial glide and neuromuscular dysfuntion.
\end{abstract}

Case description: The patient was a 71-year old female who underwent a tota shoulder replacement for treatment of significant osteoarthritis. Initially she presented with significant pain, loss of strength, range of motion (ROM) and function.

Intervention: Traditional therapeutic exercise and manual therapy was combined with TMR techniques, including contralateral arm raise, straight leg raise and spine twist, to control pain and allow the patient earlier achievement of shoulder ROM.

Outcome: Subjective report included decreased pain and improved function assessed with the QuickDASH, as well as satisfaction with the rapidity of recovery. Improved passive range of motion (PROM), active range of motion (AROM), posture and strength was also recorded at discharge.

Discussion: A combination of traditional intervention and TMR techniques were successful in controlling pain early in rehabilitation, as well as restoring function and ROM. This case study supports the use of TMR during TSA rehabilitation.

Keywords: total shoulder arthroplasty, rehabilitation, physical therapy, total motion release
Volume 2 Issue 3 - 2018

Jennifer Adame Walker

Assistant Professor, Department of Physical Therapy, California State University Fresno, USA

Correspondence: Jennifer Adame Walker,Assistant Professor, Department of Physical Therapy, California State University Fresno, USA, Tel 559355 930I, Fax 559278 3635.

Email jadame@mail.fresnostate.edu

Received: May 04, 2018 | Published: May 22 , 2018

\section{Introduction}

The incidence of shoulder replacement has increased significantly in the United States in the past 10 years, becoming the $3^{\text {rd }}$ most performed total joint arthroplasty after hip and knee. ${ }^{1-3}$ Total shoulder arthroplasty (TSA) surgeries have increased by approximately 3000 cases every year for the past 12 years in the US. ${ }^{4}$ This increase in occurrence has led to changes in both implant design and surgical techniques ${ }^{5}$ as well as increased referral for postoperative rehabilitation. There is a large body of evidence describing surgical technique and implant design; however, specific postoperative rehabilitation contribution is lacking. ${ }^{5}$ Many rehabilitation studies are based on the original protocol developed by Neer and Hughes in 1975, with progression based on timelines rather than subjective or objective data. A systematic review by Kraus et al revealed that most rehabilitation protocols were broken into 3 or 4 phase models but differed greatly on when to initiate treatment, allowable range of motion (ROM) and when to initiate strengthening. ${ }^{3}$ While it is clear that appropriate post-operative rehabilitation is crucial in the return to full functional status, it is unclear what the most appropriate or beneficial protocol is for patients following total or partial shoulder repair.

The most common indication for TSA is degenerative joint disease or osteoarthritis. Other pathologies indicating TSA include rheumatoid arthritis, avascular necrosis and traumatic fracture. ${ }^{5}$ Often the patient undergoing TSA is over 65 years of age with chronic shoulder pathology ${ }^{6}$ or symptoms that have not significantly decreased with conservative treatment. These patients have often had dysfunction and pain for years prior to undergoing TSA and have resultant weakness and postural imbalance. These surgical procedures are becoming more prevalent, as well as more successful in decreasing patient reported pain and improving function in patients with degenerative joint disease. ${ }^{7-10}$ With the increasing aging population and the increased level of activity amongst those seniors, it is likely that the incidence of TSA will continue to increase exponentially.

Appropriate shoulder function depends on a balance of strength, stability and available motion. ${ }^{7}$ The anatomical design of the shoulder is complex and surgery further alters the biomechanical complexity. TSA involves a surgical approach through the deltoid and pectoralis major to expose the glenohumoral joint. The biceps, coracobrachialis and pectoralis minor are retracted, the coracoacromial ligament is released as is the subscapularis, ${ }^{5}$ pectoralis major and lattisimus dorsi. ${ }^{6}$ Metal implants replace the surface of the humeral head and the glenoid fossa. After securing the metal implants, the tendons that were released are repaired and the incision closed. Protection of the healing subscapularis tendon is crucial during rehabilitation. ${ }^{5,6}$ Much of the published literature involving TSA focuses on preoperative 
tissue status, implant design and surgical technique, rather than post-operative rehabilitation protocol. It is clear that post-operative rehabilitation is pivotal in determining successful outcome following TSA. Charles Neer II is quoted as saying "Shoulder replacement will fail without adequate rehabilitation." 11 There are a multitude of exercises and treatment methods that may accomplish the goals of shoulder rehabilitation. One recently developed method of general rehabilitation, Total Motion Release, may be beneficial in restoring normal ROM, decreasing pain and restoring full function to patients following TSA.

TMR is based on the concept of contralateral movement. The body is divided into 4 quadrants. Dysfunction in the upper right quadrant may be addressed by movement in the upper left as contralateral in the horizontal plane, lower right as contralateral in the vertical plane, or lower left as contralateral in the diagonal plane. The method requires assessment of 6 basic movements (Figure 1) and treatment is determined by which of the movements are more out of balance from side to side. Patients grade their movement on a scale of 1-100 including a combination of pain, range, ease and speed of motion. The area with the highest score is treated first until no more improvement is noted. Treatment is most often to the side of ease (lower score) and may be repetitions or static holds into end range. TMR is based on fascial glide and neuromuscular dysfunction. Loss of normal neurodynamics, or the inability of neural tissue to glide or tension without symptom can lead to pain, decreased mobility and function. ${ }^{12}$ Exercise to the contralateral region has been shown to reverse these dysfunctions and restore pain free movement. ${ }^{12-16}$ Several studies have shown good outcomes in function of the involved limb when the uninvolved limb is exercised. However, there is no known published literature including TMR in a post-surgical rehabilitation protocol.

The purpose of this case report is to describe the post-surgical rehabilitation of a 71-year old female following TSA, using TMR techniques in combination with traditional physical therapy procedures. A 71-year old right handed female with long standing shoulder pain, resistant to conservative treatment underwent a left TSA. She was referred to physical therapy 3 weeks later. She was living in a two story home with her husband. Both she and her husband are retired, in good health and independent in all activities of daily living (ADLs). She stated she is walking 4-5 times per week for exercise. The patient reported prior to surgery she enjoyed quilting, playing with her grandchildren, socializing with friends and traveling. Upon arrival the patient was in good spirits but concerned as she felt she may have re-injured her left shoulder 2 weeks after surgery when she attempted to raise her arm above shoulder height. Consultation with the physician's assistant indicated the structures were still intact. The patient was not wearing her prescribed sling and had discontinued use of pain medication due to constipation problems. She complained of achy pain to her left shoulder, upper arm, neck and upper back averaging $4 / 10$. She reported pain of $0 / 10$ at rest and 10/10 when she raised arm above shoulder height. ${ }^{17}$ Prior to the surgery, she states her pain was a constant $6 / 10$ and limited her from completing her ADLs and recreational activity. She denied any numbness/tingling into the arm or hand and no pain below the elbow. The patient stated she was unable to sew/quilt and cook. She required her husband's assistance for showering, dressing and drying her hair. She stated there was no significant change in her sleep habits; however she did have increased achy pain in the morning upon waking.

Her past medical history includes a left thumb reconstruction, hysterectomy, cholecystectomy, right thumb reconstruction, bilateral
ACL reconstruction, right shoulder decompression, right cataract surgery and a left SLAP repair. Medications included Lipitor, a baby aspirin, an over the counter probiotic, Celebrex, Prilosec and Synthroid. A systems review determined the neuromuscular and integumentary systems were involved. From her medication list, her endocrine and gastrointestinal systems appear implicated but would not likely affect treatment. The cardiopulmonary system was managed with medication and patient reported no symptoms. The significant limitations in her daily and recreational activities would likely impact her psychosocial health and should be continually monitored. ${ }^{18,19}$ The patient's primary goal was to return to her prior level of function including independent ADLs, driving and caring for her grandchildren. Another of her goals was to be able to participate in a quilting retreat 3 months after surgery.

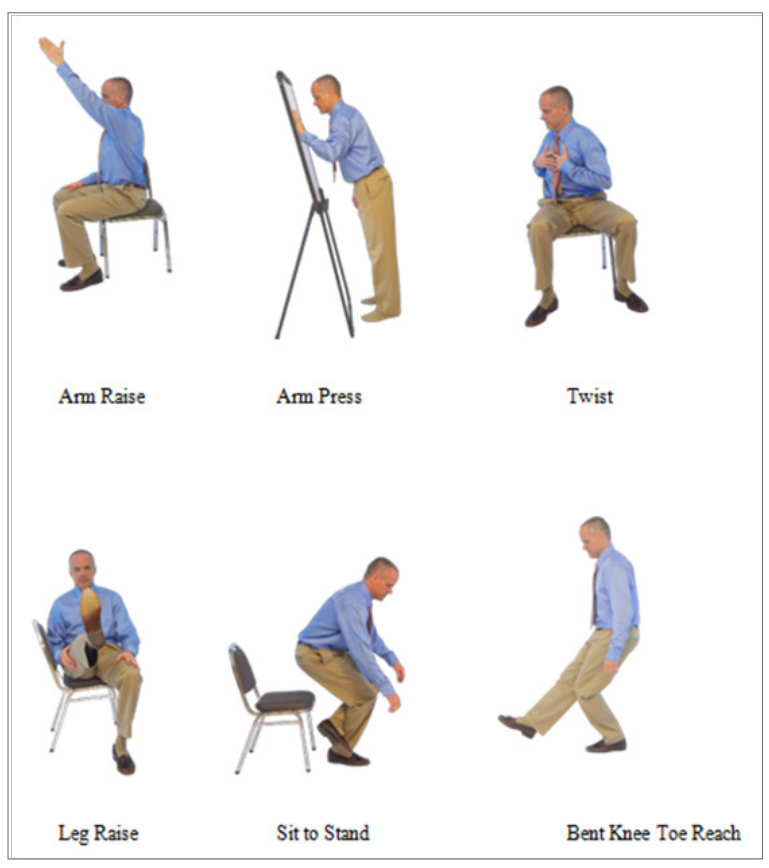

Figure I The FAB 6.

Table I Tests and measures

\begin{tabular}{|c|c|c|c|c|}
\hline \multirow{4}{*}{$\begin{array}{l}\text { Numeric pain } \\
\text { rating scale }\end{array}$} & \multicolumn{2}{|c|}{$\begin{array}{l}\text { Initial evaluation } \\
\text { results (week } 0 / \\
\text { post surg week } 3 \text { ) }\end{array}$} & \multicolumn{2}{|c|}{$\begin{array}{l}\text { Final evaluation } \\
\text { (week 8/post surg } \\
\text { week I2) }\end{array}$} \\
\hline & \multicolumn{2}{|c|}{ Rest: 0/10 } & \multicolumn{2}{|c|}{ Rest: $0 / 10$} \\
\hline & \multicolumn{2}{|c|}{ Worst: I0/10 } & \multicolumn{2}{|c|}{ Worst: $1 / 10$} \\
\hline & \multicolumn{2}{|c|}{ Current: $4 / 10$} & \multicolumn{2}{|c|}{ Current: $0 / 10$} \\
\hline AROM & Right & Left & Right & Left \\
\hline Shoulder flexion & 170 & 70 & 170 & 140 \\
\hline Extension & 70 & 50 & 72 & 65 \\
\hline Abduction & 180 & 70 & 178 & 120 \\
\hline Internal rotation & 40 & 15 & 45 & 30 \\
\hline External rotation & 80 & 15 & 75 & 20 \\
\hline
\end{tabular}


Table continuded

\begin{tabular}{lllll}
\hline $\begin{array}{l}\text { Numeric pain } \\
\text { rating scale }\end{array}$ & $\begin{array}{l}\text { Initial evaluation } \\
\text { results (week 0/ } \\
\text { post surg week 3) }\end{array}$ & $\begin{array}{l}\text { Final evaluation } \\
\text { (week 8/post surg } \\
\text { week I2) }\end{array}$ \\
\hline MMT & $4+/ 5$ & Deferred & $4+/ 5$ & $4 / 5$ \\
Flexion & $5 / 5$ & Deferred & $5 / 5$ & $4 / 5$ \\
Extension & $4 / 5$ & Deferred & $4 / 5$ & $4 / 5$ \\
Abduction & $4 / 5$ & Deferred & $4 / 5$ & $4 / 5$ \\
Internal rotation & $4 / 5$ & Deferred & $4 / 5$ & $4 / 5$ \\
External rotation & $47.3 \%$ disabled & $18.2 \%$ disabled \\
DASH & \multicolumn{4}{l}{} \\
\hline
\end{tabular}

\section{Clinical impression I}

The primary impairment for this patient appeared to be decreased upper extremity (UE) function following surgical joint replacement. The patient reported requiring assistance with all ADLs including grooming, dressing and transportation and fixing meals. The plan for initial examination focused on AROM to assess the patient's willingness to move and pain levels with activity. PROM and manual muscle testing (MMT) would be assessed as tolerated by the patient. Although it was not expected, protected ROM would be a precaution during the examination. Postural assessment would also be a priority to optimize the patient's potential for success. Lastly, TMR ${ }^{\circledR}$ assessment of the healthy joints would be assessed for potential use in the plan of care.

\section{Examination}

Upon inspection the patient was found to have a mild forward head, rounded shoulders bilaterally with moderate guarding of the left shoulder and upper extremity. The incision was well healed with minimal puckering indicating subcutaneous adhesion. There was a minimal increase in temperature of the left shoulder as compared to the right but no sign of infection or drainage from the incision. The left shoulder appeared to be mildly down sloping from the base of the neck to the acromioclavicular (AC) joint. The primary focus of the examination was functional capacity of the patient's left upper extremity. According to her self-reported disability index using the Quick DASH, the patient was $77.3 \%$ disabled. The Quick DASH asks patients to rate their level of disability from no difficulty to unable to perform for a variety of tasks including sleep, grooming, pain and carrying objects. ${ }^{20}$ Following initial observation and selfassessment of disability, AROM of the left shoulder was assessed using standard positioning and goniometer alignment and was found to be significantly limited and painful as noted in Table 1. PROM was deferred due to increased pain in left shoulder. Cervical ROM was within normal limits with complaints of contralateral tension in side bending and bilateral tension in flexion. Manual muscle testing of the right upper extremities was assessed according to Daniel's and Worthingham's guidelines. ${ }^{21,22}$ Strength testing of the left upper extremity was deferred. The patient was independent in transfers including sit $\leftarrow \rightarrow$ stand, bed mobility and gait, however left UE motion was guarded with these activities. The patient's vitals were assessed prior to and following light activity with no reading out of the normal parameters.

Palpation indicated increased muscular tension and tenderness to upper trapezius, levator scapulae and suboccipitals, left $>$ right.
Further active testing using TMR protocols indicated imbalance for arm raise (left affected) and leg raise (right affected). Re-assessment of AROM of left shoulder elevation and subjective pain response was improved on the left shoulder following treatment of the right shoulder, indicating a positive response to TMR protocol.

\section{Clinical Impression 2}

The patient was referred to physical therapy following left shoulder replacement. Physical examination confirmed the diagnosis and expected impairments; however, a number of other factors appeared to be contributing to the patient's functional limitations. Her history of surgical repairs and reconstructions lends evidence to an overall laxity and poor motor control for this patient. Furthermore, her postural dysfunction increased her risk for shoulder impingement and dysfunctional movement patterns throughout her ADLs. Lastly, the patient responded well to TMR principles during evaluation. Therefore, those exercises would likely benefit her in addition to standard rehabilitation protocols.

\section{Interventions}

The patient was seen $2 \mathrm{x} /$ week for 8 weeks for a total of 16 visits. The main focus early in post-surgical rehabilitation is to control pain and restore normal ROM. ${ }^{23}$ At the initial evaluation it was noted that the patient had poor posture, down sloping shoulder on the surgical side and constant, low level pain. The patient was advised to return to wearing her sling for 3-4 hours at a time to provide more support for the healing joint. She was also educated on proper upright positioning, including decreased thoracic kyphosis, scapular retraction to a more neutral position and appropriate head placement with the ear aligned with the AC. The patient was able to achieve this position without complaint but required moderate verbal cues to maintain the position throughout the initial treatment session.

A hot pack was applied to the left shoulder at the beginning of each session for pain control and patient comfort. ${ }^{24}$ Traditional intervention includes Codman/Pendulum exercises, Pulley exercises, Wand and Wall climbs, or any combination of these to improve ROM. Several of these exercises were initiated with this patient as shown in Table 2. During the evaluation the patient was assessed using a TMR approach. Her response was favorable in using contralateral arm raise and ipsilateral leg raise to address both pain and ROM limitations. These exercises were prescribed for 3 sets of 10 daily with a recheck of left shoulder elevation following. If subjective response did not improve the patient was advised to change to holding exercise at end range for 30 seconds for 3 sets. If subjective response did improve, the patient was advised to continue for up to 3 sets. In addition to ROM exercises for the left shoulder, cervical stretches were prescribed to address muscular tension resulting from guarding against pain. Manual therapy was performed following exercises to decrease tension in both upper trapezii, cervical paraspinals and sub occipital muscles. ${ }^{25}$ Soft tissue mobilization, skin rolling, strumming and sub occipital release were combined with good results. The patient consistently reported decreased tension following manual techniques. Lastly, ice was applied to the left shoulder at the completion of treatment for pain and edema control.

The patient was cleared by her surgeon to initiate AROM and light resistance training 4 weeks after the initial evaluation, 7-weeks postsurgery. At this time AAROM was initiated in the form of wall climbs for both flexion and abduction. The patient reported a significant 
increase in pain following the addition of these exercises so she was advised to decrease the height of elevation to $50 \%$. The patient was re-evaluated for the FAB 6 exercises in TMR and arm elevation and leg raise remained the most imbalanced. The patient reported success with the 3 sets of 10 reps. Therefore, that exercise was not changed. Basic isometric exercises (wall push) were also initiated in flexion, abduction and extension. The extension exercise further assisted in the patient's increased tolerance for appropriate posture. The patient tolerated the addition of active assisted range of motion (AAROM) and isometric training well and was able to progress to light resistive training the following week. Theraband exercises including shoulder flexion, extension and rhomboid row were added in week 5 of PT. In addition, isometric exercise for internal and external rotation using the Theraband was added with continued postural cueing. The following week the patient was able to progress to AROM including flexion, scaption and abduction to 90 degrees as well as extension to 40 degrees. The final 2 weeks of treatment consisted of continuing her AAROM, AROM and Theraband isometric exercises for rotation. TMR techniques were continued throughout rehabilitation and the patient was educated on how to use the technique for self- treatment following discharge. Treatment progression can be found in Table 2.

Table 2 Treatment progression

\begin{tabular}{|c|c|c|c|}
\hline Phase & $\begin{array}{l}\text { PT visit/weeks post- } \\
\text { surgery }\end{array}$ & Intervention & Sets/reps \\
\hline \multirow{10}{*}{ I } & \multirow{8}{*}{ I / 3 weeks post } & Hot pack to left shoulder & 15 minutes \\
\hline & & Postural education & \\
\hline & & TMR® exercise & Right arm elevation $3 \times 10$ Left leg elevation seated $3 \times 10$ \\
\hline & & $\begin{array}{l}\text { STM UT, LS, CPS, SO Scar } \\
\text { mobilization }\end{array}$ & 10 minutes \\
\hline & & & Codman/pendulum $3 \times 10$ Shrugs $3 \times 10$ \\
\hline & & HEP instruction & Scap squeeze $3 \times 10$ \\
\hline & & & $\begin{array}{l}\text { Cervical stretches for flexion, } \\
30 \text { seconds each }\end{array}$ \\
\hline & & Manual therapy & PROM flexion, scaption $\times 8$ minutes \\
\hline & \multirow{2}{*}{ 2-8/ 4-6 weeks post } & All of the above & Pulleys flexion, scaption 30 each \\
\hline & & Added PROM & \\
\hline \multirow{4}{*}{2} & \multirow[t]{2}{*}{ 9/ 7 weeks post } & All of the above & $\begin{array}{l}\text { Wall press in flexion, abduction, extension (all with elbow } \\
\text { bent } 90 \text { degrees) }\end{array}$ \\
\hline & & Added isometric & $3 \times 10$ each \\
\hline & \multirow{2}{*}{10} & All of the above & Flexion $\times 10$ \\
\hline & & Added wall climb & Abduction $\times 10$ \\
\hline \multirow{5}{*}{3} & \multirow{3}{*}{ II-|4/8-II weeks post } & All of the above & $\begin{array}{l}\text { T-band isometric for flexion, extension, internal and external } \\
\text { rotation }\end{array}$ \\
\hline & & Added T-band isometric & Rhomboid rows with t-band $3 \times 10$ \\
\hline & & & *modified wall climbs to max height of 90 degrees due to pain \\
\hline & \multirow{2}{*}{$15-16 / 12$ weeks post } & All of the above & $\begin{array}{l}\text { T-band flexion to } 90 \text { degrees, extension as tolerated, internal } \\
\text { rotation to } 70 \text { degrees (elbow at side), external rotation to } 70 \\
\text { degrees (elbow at side) }\end{array}$ \\
\hline & & $\begin{array}{l}\text { Added AROM with t-band } \\
\text { resistance }\end{array}$ & $\begin{array}{l}\text { Continued to progress height of wall climbs to full ROM in } \\
\text { flexion and abduction }\end{array}$ \\
\hline
\end{tabular}

Abbreviations: STM, soft tissue massage; UT, upper trapezii; LS, levator scapula; SO, suboccipitals; CPS, cervical paraspinals.

\section{Results}

At discharge the patient achieved full PROM in flexion, extension and abduction. Her AROM was significantly improved as listed in Table 1. She reported independent performance of ADLs with mild limitations due to muscular fatigue. She was able to attend her quilting retreat and had returned to babysitting her grandchildren for short periods of time. The patient reported a decrease in disability from $77 \%$ originally to $18 \%$ at discharge as measured by the Quick DASH. Her primary limitations were activity that required significant strength (pressing on a rolling cutter) and/or time (holding a hair dryer). The patient's posture was improved by approximately $75 \%$ and she required only minimal cueing to return to neutral. However, 
she continued to present with an increased thoracic kyphosis and mild forward head. The patient was educated on the benefits of appropriate posture, not only for use of her shoulder, but to avoid compression fractures that are common in osteoporetic women. The patient was able to verbally describe and physically demonstrate the TMR exercises (arm raise and leg raise) that would be used following discharge for self-management along with continued performance of her home exercise program. Overall, the patient made excellent progress and attained a functional level exceeding her expectations. The addition of the TMR exercises may have played a role in the successful timeframe of her recovery and should provide continued relief in the future.

\section{Discussion}

The purpose of this case report was to describe a post TSA rehabilitation protocol including both traditional physical therapy, as well as TMR ${ }^{\circledR}$ techniques. As previously noted, there is a large body of literature to describe and support pre-surgical tissue health, surgical techniques and implant design, but very little evidence to support post-surgical rehabilitation approach. In fact, one study by Mulieri found no significant difference in outcomes between a traditional PT rehabilitation and a surgeon supervised home program following TSA. ${ }^{26}$ There were several limitations to this study however and the majority of expert testimony does recognize the need for post-surgical rehabilitation for successful return to activity. This patient made good progress in her rehabilitation and met her goals in a timely manner, giving support to the need for formal physical therapy services. A report by Salamh and Speer outline the typical progression of post TSA rehabilitation, indicating 3 phases. Phase 1 (3 weeks after surgery) includes sling wearing and no PT but AROM to elbow, wrist and hand. Phase 2 (weeks 4-7) is when PT is initiated with emphasis on protecting musculotendinous repair. PROM and AAROM are performed in Phase 2. Phase 3 (weeks 8-12) allow for full PROM/AROM. Lastly, Phase 4 (after 12 weeks) allows progression into resistive exercise. This is the point at which most patients are discharged from formal PT and encouraged to continue with a home program. ${ }^{6}$

The progression of rehab for this patient followed the above guidelines. The authors mention that full PROM and AROM are not achieved until 6 months; however this patient had full PROM into flexion, extension and abduction at 3 months. She also reported lower levels of pain during treatment which may have been influenced by the addition of TMR protocols. While there is no literature discussing the use of TMR principles with shoulder rehabilitation, one study by Baker et al describes the positive outcome of a TMR program in reversing chronic hamstring tightness in a speed walker. ${ }^{16}$ The patient had experienced chronic pain and extensibility dysfunction in both posterior legs. Use of TMR technique increased her sit and reach by 5 $\mathrm{cm}$ and her straight leg raise by 31.5 degrees in 1 week. At discharge the patient had a negative 90/90 active knee extension, active straight leg raise, slump test and complete resolution of symptoms. The findings persisted at follow up 1 and 3 months later. ${ }^{16}$ Another study by Lepley et al reported increased quadriceps strength in a similar manner. Participants were tasked to exercise one limb eccentrically for 8 weeks and the other not at all. There was a significant increase in the quadriceps strength of the unexercised limb leading researchers to propose increased strength due to enhanced neural activity. ${ }^{27}$ The addition of TMR ${ }^{\circledR}$ exercises may have provided improved outcomes in a shorter period of time in this case. The patient's recovery is consistent with outcomes published in the literature, specifically pain reduction, increased ROM and function. ${ }^{28-34}$ Again, there is little material in the literature regarding specific protocol for post TSA rehabilitation. This case study lends evidence to the 4 Phase protocol with the addition of TMR $®$ techniques for pain management, ROM and functional gains. ${ }^{35-40}$

\section{Conclusion}

While there is an abundance of literature pertaining to Total Shoulder Arthroscopy, little of it is in relation to post-surgical rehabilitation. This case demonstrates the positive outcome associated with post-surgical rehabilitation. The initial concern in this case was to control pain and provide a protected environment in which to allow the tissue to heal. This was accomplished through use of sling, gentle ROM and postural education. As the tissue healing progressed, additional techniques, including TMR, allowed a return to full functional status. The addition of the TMR techniques was simple and patient education of these techniques will allow her to manage her condition in the future. ${ }^{41}$

\section{Acknowledgements}

Patient consent was obtained at time of treatment.

\section{Conflict of interest}

Author declares that there is no conflict of interest.

\section{References}

1. Zarkadas P, Throckmorton T, Dahm DL, et al. Patient reported activities after shoulder replacement and hemiarthroplasty. J Shoulder Elbow Surg. 2011;20(2):273-280.

2. Day TS, Lau E, Ong KL, et al. Prevalence and projections of total shoulder and elbow arthroplasty in the United States to 2015. J Shoulder Elbow Surg. 2010;19(8):1115-1120.

3. Kraus M, Krischak G, Tepohl L. Postoperative Rehabilitation of Patients with Shoulder Arthroscopy: A Review on the Standard of Care. Int $J$ of Phys and Med Rehabil. 2014;S5:001.

4. Kim SH, Wise BL, Zhang Y, et al. Increasing Incidence of Shoulder Arthroplasty in the United States. J Bone Joint Surg Am. 2011;93(24):2249-54.

5. Wolff AL, Rosenzweg L. Anatomical and biomechanical framework for shoulder arthroplasty rehabilitation. J Hand Ther. 2017;30(2):167-174.

6. Salamh PA, Speer KP. Post-Rehabiliation Exercise Considerations Following Total Shoulder Arthroplasty. Strength and Conditioning Journal. 2013;35(4):56-63.

7. Wilcox RB, Arslanian LE, Millett PJ. Rehabilitation Following Total Shoulder Arthroplasty. J Orth and Sports Phys Ther. 2005;35(12):821-836.

8. Puskas B, Harreld K, Clark R, et al. Isometric strength, range of motion and impairment before and after total and reverse shoulder arthroplasty. $J$ Shoulder Elbow Surg. 2013;22(7):869-876.

9. Bryant D, Litchfield R, Sandow M, et al. Comparison of Pain, Strength, Range of Motion, and Functional Outcomes after Hemiarthroplasty and Total Shoulder Arthroplasty in Patient with Osteoarthritis of the Shoulder. J Bone Joint Surg Am. 2005;87(9):1947-1956.

10. Norris TR, Iannotti JP. Functional outcome after shoulder arthroplasty for primary osteoarthritis. A multicenter study. J Shoulder Elbow Surg. 2002;11(2):130-135. 
11. Brems JJ. Rehabilitation following shoulder arthroplasty. In: Friedman RJ, editor. Arthroplasty of the Shoulder. New York: Theime Medical Publishers; 1994. p. 99-111.

12. Baker R, Nasypany AM, Seegmiller JG. Reversal of Chronic Musculoskeletal Dysfunction and Pain.

13. Fermin SL, Larkins L, Beene SN, et al. The Effect of Contralateral Exercise on Patient Pain and Range of Motion. Journal of Sport Rehabilitation. 2018;27(2):185-188.

14. Lee M, Gandevia SC, Carroll TJ. Unilateral strength training increases voluntary activation of the untrained limb. Clin Neurophysiol. 2009;120(4):802-808

15. Huang HJ, Ferris DP. Upper and Lower Limb Muscle Activation is Bidirectionally and Ipsilaterally Coupled. Med Sci Sports Exerc. 2009;41(9):1778-1789.

16. Baker RT, Hansberger B1, Warren L, et al. A Novel Approach for the Reversal of Chronic Apparent Hamstring Tightness: A Case Report. Int J Sports Ther. 2015;10(5):723-733.

17. Rehab Measures: Numeric Pain Rating Scale. 2017.

18. Warner J. Changes in Psychological Status and Health Related Quality of Life Following Total Shoulder Arthroplasty. J Bone Joint Surg Am. 2017;99(12):1030-1035.

19. Werner BC, Wong AC, Chang B, et al. Depression and Patient-Reported Outcomes Following Total Shoulder Arthroplasty. J Bone Joint Surg Am. 2017;99(8):688-695.

20. MacDermid JC, Khadilkar L, Birmingham BB, et al. Validity of the QuickDASH in Patients with Shoulder Related Disorders Undergoing Surgery. $J$ Ortho Sports Phys Ther. 2015;45(1):25-36.

21. Rehab Measures: Manual Muscle Test. Rehabilitation Institute of Chicago; 2017.

22. Helen J. Hislop DA, Marybeth Brown. Daniels and Worthingham's Muscle Testing: Techniques of Examination and Performance Testing 9th ed. Missouri: St. Louis, Elsevier Inc.

23. Denard PJ, Ladermann A. Immediate versus delayed passive range of motion following total shoulder arthroplasty. J Shoulder Elbow Surg. 2016;25(12):1918-1924.

24. Halsell M. Moist Heat for Relief of Postoperative Pain. The American Journal of Nursing. 1967;67(4):767-770.

25. Dolder VDP, Roberts L. A trial into the effectiveness of soft tissue massage in the treatment of shoulder pain. Aust J Physiother. 2003;49(3):183-188.

26. Mulieri PJ, Holcomb JA, Dunning P, et al. Is a formal physical therapy program necessary after total shoulder arthroplasty for osteoarthritis? $J$ Shoulder Elbow Surg. 2010;19(4):570-579.
27. Lepley LK, Smith PRM. Cross-Education Strength and Activation after Eccentric Exercise. J Athl Train. 2014;49(5):582-589.

28. Razmjou H, Stratford P, Kennedy D, et al. Pattern of recovery following total shoulder arthroplasty and humeral head replacement. BMC Musculoskeletal Disord. 2014;15:306.

29. Carter MJ, Mikuls TR, Nayak S, et al. Impact of total shoulder arthroplasty on generic and shoulder-specific health-related quality of life measures: a systematic literature review and meta-analysis. J Bone Joint Surg Am. 2012;94(17):e127.

30. Fevang BT, Lygre SH, Bertelsen G, et al. Good function after shoulder arthroplasty. Acta Orthop. 2012;83(5):467-473.

31. Deshmukh AV, Korris M, Zurakowski D, et al. Total shoulder arthroplasty: long term survivorship, functional outcome and quality of life. J Shoulder Elbow Surg. 2003;14(5):471-9.

32. Edwards TB, Kadakia NR, Boulahia A, et al. A comparison of hemiarthroplasty and total shoulder arthroplasty in the treatment of primary glenohumoral osteoarthritis: results of a multicenter study. J Shoulder Elbow Surg. 2003;12(3):207-213.

33. Torchia ME, Cofield RH, Settergren CR. Total shoulder arthroplasty with the Neer prosthesis: long term results. J Shoulder Elbow Surg. 1997;6(6):495-505.

34. Brown D, Friedman RJ. Postoperative Rehabilitation Following Total Shoulder Arthroplasty. Orthop Clin North Am. 1998;29(3):535-547.

35. Morris T, Newby NA, Wininger M, et al. Inter-limb transfer of learned ankle movements. Exp Brain Res. 2009;192(1):33-42.

36. Neer CS. Replacement Arthroplasty for Glenohumoral Osteoarthritis. $J$ Bone and Joint Surg. 1974;56(1):1-13.

37. Wright T, Easley T, Bennett J, et al. Shoulder arthroplasty and its effect on strain in the subscapularis muscle. Clin Biomech. 2015;30(4):373-376.

38. Sciascia AD, Morris BJ, Jacobs CA, et al. Using Patient-Reported Outcome Measures to Determine Satisfaction following Anatomic Shoulder Arthroplasty. J Shoulder Elbow Surg. 2017;26(5):e154.

39. Galvin JW, Eichinger JK, Boykin RE, et al. Posterior shoulder instability following anatomic total shoulder arthroplasty: A case report and review of management. Int J Shoulder Surg. 2015;9(4):131-134.

40. Teusink MJ, Virani NA, Plikandriotis JA, et al. Cost Analysis in Shoulder Arthroplasty Surgery. Adv Orthop. 2012;1-4.

41. Baker DT. TMR Seminars. 2017. 